\title{
Ductile and Brittle Yielding in Thermal and Athermal Amorphous Materials
}

\author{
Hugh J. Barlow, James O. Cochran $\odot$, and Suzanne M. Fielding \\ Department of Physics, Durham University, Science Laboratories, South Road, Durham DH1 3LE, United Kingdom
}

(Received 16 December 2019; revised 22 May 2020; accepted 9 September 2020; published 15 October 2020)

\begin{abstract}
We study theoretically the yielding of sheared amorphous materials as a function of increasing levels of initial sample annealing prior to shear, in three widely used constitutive models and three widely studied annealing protocols. In thermal systems we find a gradual progression, with increasing annealing, from smoothly "ductile" yielding, in which the sample remains homogeneous, to abruptly "brittle" yielding, in which it becomes strongly shear banded. This progression arises from an increase with annealing in the size of an overshoot in the underlying stress-strain curve for homogeneous shear, which causes a shear banding instability that becomes more severe with increasing annealing. Ductile and brittle yielding thereby emerge as two limiting cases of a continuum of yielding transitions, from gradual to catastrophic. In contrast, athermal systems with a stress overshoot always show brittle yielding at low shear rates, however small the overshoot.
\end{abstract}

DOI: $10.1103 /$ PhysRevLett.125.168003

Amorphous materials include soft glasses such as dense colloids, emulsions, foams, and microgels [1-5], as well as hard molecular and metallic glasses [6,7]. Under low loads or small deformations, such materials show solidlike behavior. Under higher loads or larger deformations, they yield plastically. For some systems, the dynamical process whereby an initially solidlike sample yields to give a finally fluidized flow is smooth and gradual [8-16]. Others yield abruptly, showing catastrophic failure [17]. For both "ductile" materials, which yield smoothly and gradually, and "brittle" materials, which yield abruptly and catastrophically, understanding the statistical physics of yielding is the focus of intense interest. Theories have been put forward based on a first order transition in a replica theory [18,19]; a critical point [20,21]; a directed percolation transition [22,23]; and a spinodal [24-28]. Microscopic precursors to yielding have recently been observed in soft materials [29-31].

Recent mean field calculations [32,33] have suggested that the underlying stress-strain relation $\Sigma(\gamma)$ for an athermal amorphous material undergoing quasistatic shear displays a qualitative change in form from the lower to the upper curve in Fig. 1(a) as the degree to which a sample is annealed prior to shear increases. Poorly annealed samples (lower curve) then yield in a smoothly ductile way. Well annealed samples (upper curve) instead show catastrophic brittle yielding, as the stress drops precipitously once the overhang is reached. In this scenario, ductile and brittle

Published by the American Physical Society under the terms of the Creative Commons Attribution 4.0 International license. Further distribution of this work must maintain attribution to the author(s) and the published article's title, journal citation, and DOI. yielding are separated by a random critical point, at which the stress-strain curve switches between two qualitatively different shapes with increasing annealing. Particle simulations were argued to agree with this scenario [32].

Here, we propose an alternative scenario, in which the underlying stress-strain curve for homogeneous shear has an overshoot, not an overhang, followed by a regime of negative slope $\partial_{\gamma} \Sigma<0$. In thermal systems such as colloidal glasses, where $k_{B} T$ is important compared to barriers for particle rearrangements, a state of initially homogeneous shear becomes linearly unstable to the formation of shear bands in this negatively sloping regime [34-36], with the severity of banding (defined below) after an (adimensionalized) stress drop of magnitude $\Delta \Sigma$ from the stress maximum scaling as $\exp (\Delta \Sigma)$. A large stress drop (strong annealing) thus causes severe banding and brittle failure, whereas a small stress drop (weak annealing) causes only weak banding, and failure remains ductile; Fig. 1(b). In slowly sheared athermal systems, in contrast,

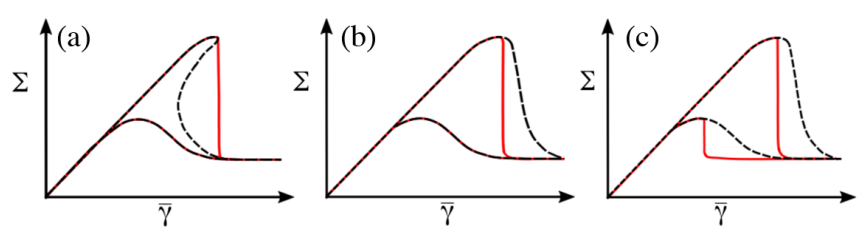

FIG. 1. Schematic shear stress vs strain in different possible scenarios for ductile and brittle yielding: as suggested (a) in Refs. [32,33], and here for (b) thermal and (c) athermal systems. In each case the upper (lower) curve is for a better (less well) annealed sample. Dashed lines: theoretical curve with homogeneous shear artificially enforced. Solid lines: precipitous drop from (a) stress overhang, or (b), (c) from homogenous curve due to shear banding, giving brittle yielding. 
the severity of banding diverges (at the level of a linear instability calculation) as the overshoot is approached as $1 / \partial_{\gamma} \Sigma$, giving brittle failure for any sized stress drop, however small; Fig. 1(c). We substantiate these scenarios both analytically and numerically in a thermal fluidity model [37] and an athermal elastoplastic model [38]; and numerically [39] in the soft glassy rheology model [40] in both thermal and athermal regimes. Crucially, in neither thermal nor athermal scenario does a critical point of the kind in Fig. 1(a) separate ductile and brittle yielding.

For consistency with the vocabulary of Ref. [32], we use the term brittle to characterize abrupt yielding in which the rate of failure is much larger than the rate of the imposed deformation, and in which the strain becomes strongly localized within the sample, but with a caveat that we quote from [32]: "Although this phenomenon is not accompanied by the formation of regions of vacuum, as it happens in the fracture of brittle materials, the macroscopic avalanche taking place at the discontinuous yielding transition does resemble a crack induced by a brittle fracture." Indeed, our calculations and those of Ref. [32] are performed at fixed volume, disallowing the opening of an air gap. We suggest, however, that the formation of a severe high shear band in abrupt yielding will, in studies at fixed pressure, indeed lead to the rapid opening of an air gap.

We consider a sample prepared by time $t=0$ with some level of annealing (defined below) then sheared for all $t>0$ between infinite flat parallel plates at $y=0, L_{y}$ by moving the top plate at velocity $\overline{\dot{\gamma}} L_{y} \hat{\mathbf{x}}$. The shear is assumed incompressible and inertialess. As is standard practice, we restrict all velocities $v(y, t)$ to the main flow direction $\hat{\mathbf{x}}$, and gradients to $\hat{\mathbf{y}}$. The local shear rate $\dot{\gamma}(y, t)=\partial_{y} v(y, t)$ may vary across $y$ due to shear banding. The spatially average imposed shear rate $\overline{\dot{\gamma}}=\int_{0}^{L_{y}} d y \dot{\gamma}(y, t) / L_{y}$. We track only the shear component of the stress, $\Sigma_{x y}(t)=\sigma_{x y}(y, t)+\eta \dot{\gamma}(y, t)$, with an elastoplastic contribution $\sigma_{x y}(y, t)$ and a Newtonian solvent contribution of viscosity $\eta$. Force balance requires $\partial_{y} \Sigma_{x y}=0$. Hereafter, we drop the $x y$ subscript for clarity. For the dynamics of the elastoplastic stress $\sigma$, we consider three different constitutive models: a thermal continuum fluidity model [37], an athermal elastoplastic model [38], and the soft glassy rheology model [40], separately in thermal and athermal regimes [39].

Thermal systems. - As a simple model of thermal systems we consider a continuum fluidity model [37], which supposes a Maxwell-type constitutive equation:

$$
\partial_{t} \sigma(y, t)=G \dot{\gamma}-\sigma / \tau .
$$

Here, $G$ is a constant modulus and $\tau$ is a stress relaxation time, which has its own dynamics:

$$
\partial_{t} \tau(y, t)=1-\frac{|\dot{\gamma}| \tau}{1+|\dot{\gamma}| \tau_{0}}+\frac{l_{o}^{2}}{\tau_{0}} \partial_{y}^{2} \tau
$$
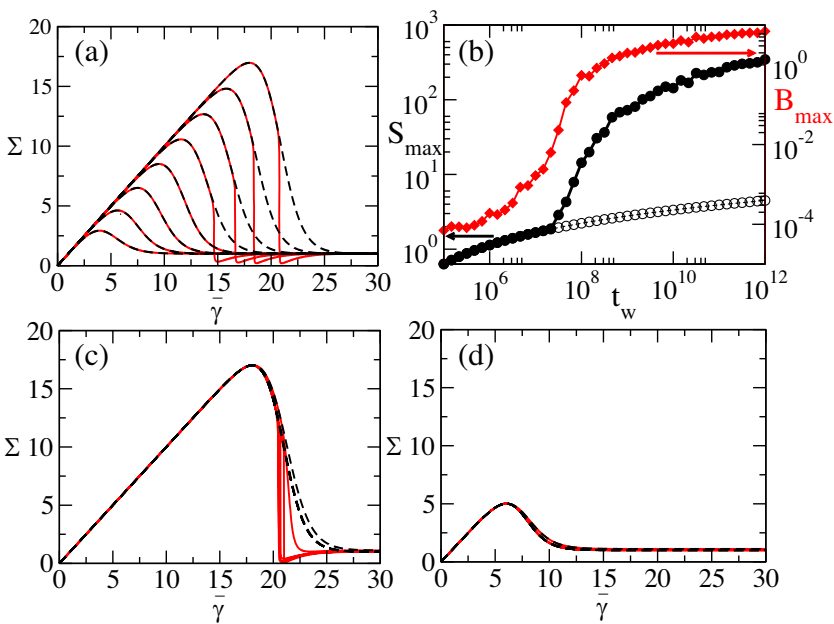

FIG. 2. (a) Stress vs strain in thermal fluidity model with homogeneous flow enforced (dashed lines) and shear banding allowed (solid lines). Imposed shear rate $\overline{\dot{\gamma}}=10^{-3}$, waiting times $t_{w}=10^{5}, 10^{6}, \ldots, 10^{12}$ in curves left to right. (b) Left vertical axis: steepest negative slope in stress-strain curve with homogeneous flow enforced (open circles) and allowing banding (closed black circles) vs sample age $t_{w}$, for fixed $\overline{\dot{\gamma}}=10^{-3}$. Right vertical axis: corresponding maximum degree of shear banding. ( $S_{\max }$ and $B_{\max }$ each averaged over 10-60 runs at each $\left.t_{w}.\right)(\mathrm{c})$ and (d) show curves in the same format as (a), but now for a fixed large stress peak $\Sigma_{\max }=17.0$ (c) or small stress peak $\Sigma_{\max }=5.0$ (d), for imposed $\overline{\dot{\gamma}}=10^{-5}, 10^{-4}, \ldots, 10^{-1}$. Steeper stress drop for smaller $\overline{\dot{\gamma}}$ in (c). $\eta=0.05, \delta=0.01 \overline{\dot{\gamma}}, l_{0}=10^{-3}$, $D t=0.01, D y=1 / 3000$.

The first term on the rhs captures aging, in which the timescale for stress relaxation increases linearly with the time $t_{w}$ for which a sample is aged before deformation commences. The second term captures rejuvenation by deformation, with $\tau_{0}$ a microscopic timescale that sets the limiting value for $\tau$ as $\dot{\gamma} \rightarrow \infty$. The mesoscopic length $l_{o}$ describes the tendency for the relaxation time of a mesoscopic region to equalize with its neighbors.

We consider a sample aged (annealed) for a time $t_{w}$ before shear commences at time $t=0$, such that $\tau(y, t=0)=t_{w}$. To seed heterogeneity, we add noise in each numerical time step $D t$ as $\sigma(y, t+D t)=\sigma(y, t)+r \delta \sqrt{D t} \cos \left(\pi y / L_{y}\right)$, with $r$ chosen from a top hat distribution between -0.5 and +0.5 , and $\delta$ small. In both this model and the athermal one below, we rescale stress, time, and length so that $G=\tau_{0}=L_{y}=1$. The solvent viscosity $\eta \ll G \tau_{0}=1$ is unimportant to the physics we describe. We use typical values $\eta=0.01-0.05$, but find no changes to our results on reducing $\eta$ further.

Figure 2(a) shows as dashed lines the stress $\Sigma(\bar{\gamma})$ as a function of the accumulating strain $\bar{\gamma}=\overline{\dot{\gamma}} t$, calculated by imposing that the shear must remain homogeneous across the sample, for several values of the sample age $t_{w}$ at a single value of the imposed shear rate $\overline{\dot{\gamma}}$. Each curve shows an initially solidlike elastic regime in which the stress 


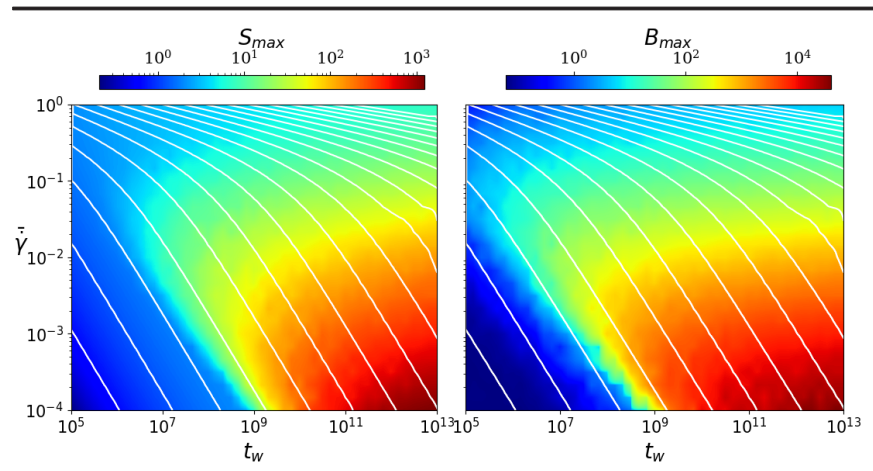

FIG. 3. Color scale showing in thermal fluidity model (left) steepest negative slope of stress versus strain $S_{\max }$ and (right) maximum degree of shear banding $B_{\max }$ during deformation. Each coordinate pair in the plane represents an average over 10-60 deformation simulations, each with an initial sample age $t_{w}$ and imposed shear rate $\overline{\dot{\gamma}}$. Contour lines show size of stress overshoot (peak minus steady state stress) $2,4,6, \ldots, 40$ (bottom left to top right). $\eta=0.05, \delta=0.01 \bar{\gamma}, l_{0}=10^{-3}, D t=0.01$, Dy $=1 / 3000$.

increases linearly with strain. At late times (large strains), the sample flows plastically with a constant value of the shear stress. For intermediate strains, the stress shows an overshoot that increases in amplitude with an increasing degree of annealing prior to shear (increasing $t_{w}$ ): an older sample shows a larger initial regime of elastic response before yielding.

We then performed separate calculations in which shear bands are allowed to form; see the solid lines in Fig. 2(a). For poorly annealed samples, each stress-strain curve still follows that of the corresponding homogeneous calculation, to good approximation: the shear field remains (nearly) homogeneous, and yielding occurs in a smoothly gradual (ductile) way. For well annealed samples, in contrast, the stress-strain curve of the heterogeneous calculation only follows that of the homogeneous one until just after the stress overshoot. It then drops precipitously as the sample becomes strongly shear banded, causing abrupt (brittle) yielding.

For each deformation simulation, defined by the values of $\left(\overline{\dot{\gamma}}, t_{w}\right)$, we quantify the abruptness of the stress drop during yielding by $S_{\max }=\max \left[-\partial_{\gamma} \Sigma\right]$, and the severity of shear banding during yielding by $B_{\max }=\max [B(\bar{\gamma})]$ with the maximization performed over all times during the deformation. [At any time $t$, or strain $\bar{\gamma}(t)$, we define the degree of shear banding $B(\bar{\gamma})$ as the maximum minus minimum local strain rate across the sample, normalized by $\overline{\dot{\gamma}}$.] These quantities are plotted as a function of sample age in Fig. 2(b), for a fixed $\overline{\dot{\gamma}}$. A regime of gradual yielding (low $S_{\max }$ ) and near homogeneous deformation (low $B_{\max }$ ) for poorly annealed samples (low $t_{w}$ ) crosses over into a regime of precipitous yielding (high $S_{\max }$ ) and strong banding (high $B_{\max }$ ) at high $t_{w}$. This crossover is explored in the full plane of $\overline{\dot{\gamma}}, t_{w}$ in Fig. 3. For $\overline{\dot{\gamma}} \ll 1$ it occurs at $\bar{\gamma} \approx 10^{m} / t_{w}$, with $m \approx 4.5$. Deviations from this scaling at higher $\overline{\dot{\gamma}}$ should be disregarded, because the model is itself only valid for $\overline{\dot{\gamma}} \ll 1$. Increasing strain localization [36] and decreasing notch fracture toughness $[41,42]$ with decreasing initial "effective temperature" have been seen in the shear transformation zone (STZ) model.

To understand these results, we perform a linear stability analysis for how strongly shear bands will form during any deformation, by writing the system's state as the sum of a time-dependent homogeneous base state (as would pertain in a theoretically idealized deformation with banding prohibited), plus an initially small heterogeneous precursor to any shear bands: $\dot{\gamma}(y, t)=\overline{\dot{\gamma}}+\delta \dot{\gamma}(t) \exp (i k y), \sigma(y, t)=$ $\bar{\sigma}(t)+\delta \sigma(t) \exp (i k y), \quad \tau(y, t)=\bar{\tau}(t)+\delta \tau(t) \exp (i k y)$. Substituting these into the model equations and expanding to first order in $(\delta \dot{\gamma}, \delta \sigma, \delta \tau)$, we find [39] the degree of shear banding $\delta \dot{\gamma}(t) / \bar{\gamma}$ after a stress drop of magnitude $\Delta \Sigma$ from the stress overshoot to scale as $\left(\delta \dot{\gamma}_{0} / \bar{\gamma}\right) \exp (\Delta \Sigma)$ in the limit $\overline{\dot{\gamma}} \rightarrow 0$, with $\delta \dot{\gamma}_{0} / \overline{\dot{\gamma}}$ the small initial heterogeneity due to noise. We further find the height of the stress overshoot to scale as an increasing function of $\bar{\gamma} t_{w}$ for low $\overline{\dot{\gamma}}$ (contours in Fig. 3). Weakly annealed systems, which have a small stress overshoot, thus remain almost homogeneous and show ductile yielding [Fig. 2(d) and dark blue regions in Figs. 3]. Strongly annealed systems, which have a large stress overshoot, show strong shear banding and brittle failure in slow shear [red region in Fig. 3 and low $\overline{\dot{\gamma}}$ curves in Fig. 2(c)]. Ductile and brittle yielding thus emerge as limiting cases of a continuum of yielding behaviors in thermal systems.

In Ref. [39], we show the same smooth crossover from ductile to brittle yielding in the soft glassy rheology model in its thermal regime, with each panel (a)-(d) in Fig. 1 of [39] strikingly analogous to its counterpart in Fig. 1.

Athermal systems.-As a simple model of an athermal amorphous material we consider an ensemble of elastoplastic elements, each corresponding to a local mesoscopic region of material [38]. In shear of rate $\dot{\gamma}$, each element builds up a local elastic shear strain $l$ as $\dot{l}=\dot{\gamma}$, giving a local shear stress $G l$, with $G$ a constant modulus. This stress is intermittently released by local plastic yielding events, which occur stochastically with rate $r(l)=\tau_{0}^{-1}$ when a local energy barrier $E$ is exceeded, $\frac{1}{2} G l^{2}>E$, and $r(l)=0$ otherwise, with $E=1$ (in our units), and $\tau_{0}=1$ a microscopic attempt time. Upon yielding, any element resets its local stress to zero. The probability distribution $P(l, t)$ of local strains obeys

$$
\dot{P}(l, t)+\dot{\gamma} \partial_{l} P=-r(l) P+Y(t) \delta(l) .
$$

Here, $Y(t)=\int d l r(l) P(l, t)$ is the ensemble average yielding rate and $\delta(l)$ is the Dirac delta function. The total elastoplastic stress $\sigma(t)=G \int d l l P(l, t)$. 
So far, we have assumed homogeneous flow in this elastoplastic model. To account for nonuniform shear, we now take $n=1, \ldots, N$ streamlines at discretized flowgradient positions $y=0, \ldots, L_{y}$, with periodic boundary conditions. The distribution $P(l, y, t)$ on any streamline then obeys Eq. (3) with $P(l, t) \rightarrow P(l, y, t), \dot{\gamma} \rightarrow \dot{\gamma}(y, t)$, and $Y(t) \rightarrow Y(y, t)$. The streamline yielding rate $Y(y, t)=$ $\int d l r(l) P(l, y, t)$ and elastoplastic stress $\sigma(y, t)=$ $G \int d l l P(l, y, t)$. Given an imposed average shear rate $\overline{\dot{\gamma}}$ across the sample as a whole, the shear rate on each streamline follows by imposing force balance: $\Sigma(t)=\sigma(y, t)+\eta \dot{\gamma}(y, t)=$ $\bar{\sigma}(t)+\eta \overline{\dot{\gamma}}$, with $\bar{\sigma}(t)=\left(1 / L_{y}\right) \int_{0}^{L_{y}} d y \sigma(y, t)$. On a timescale $\eta / G$ after any local yielding event, this recovers the $1 \mathrm{D}$ projection of the Eshelby stress propagator of 2D lattice elastoplastic models [38].

We simulate $M=80000$ elastoplastic elements on each of $N=20$ streamlines, with force balance across streamlines as described. Adjacent streamlines are further weakly coupled by adjusting the stress of three randomly chosen elements on each adjacent streamline an amount $w l(-1,+2,-1)$ following any yielding event of size $l$, with $w$ a small parameter, mimicking the stress diffusion term in Eq. (2) above. Before shear commences at time $t=0$ we assign each element an initial local strain from a Gaussian, $P_{0}(l)=\exp \left(-l^{2} / 2 \tilde{l}^{2}\right) / \sqrt{2 \pi} \tilde{l}$, with smaller $\tilde{l}$ corresponding to better annealed samples.

Figure 4(a) shows as dashed lines the stress $\Sigma(\bar{\gamma})$ as a function of strain $\bar{\gamma}=\overline{\dot{\gamma}} t$, calculated by imposing that the shear must remain homogeneous, for several levels of annealing prior to shear $1 / \tilde{l}$ at a single imposed $\overline{\dot{\gamma}}$. Each curve shows an initially solidlike elastic regime in which the stress increases linearly with strain, before the stress declines as plastic yielding sets in. More strongly annealed samples show a stronger stress overshoot. (The stress later shows a persistent oscillation known to arise in simplified homogeneous elastoplastic models [38].)

We then performed separate calculations in which shear bands can form: see the solid lines in Fig. 4(a). As the stress overshoot is reached, shear bands indeed form and the stress falls precipitously, causing brittle failure. By comparing each of Figs. 4(a), 4(c), and 4(d) with their counterparts in Fig. 2, we see an important difference between thermal and athermal systems. For thermal systems, a large enough stress overshoot is required to see brittle failure. In contrast, slowly sheared athermal systems show brittle failure however small the overshoot. (Very poorly annealed samples have no stress drop, and so show ductile deformation.) The trend to increasingly sharp failure with decreasing shear rate was seen in particle simulations of a stable glass [43].

To explore this further, Fig. 5 shows color maps of the steepness of the stress drop $S_{\max }$ and severity of shear banding [44] $B_{\max }$ as a function of the degree of annealing $1 / \tilde{l}$ and imposed shear rate $\overline{\dot{\gamma}}$. Increasing overshoot heights
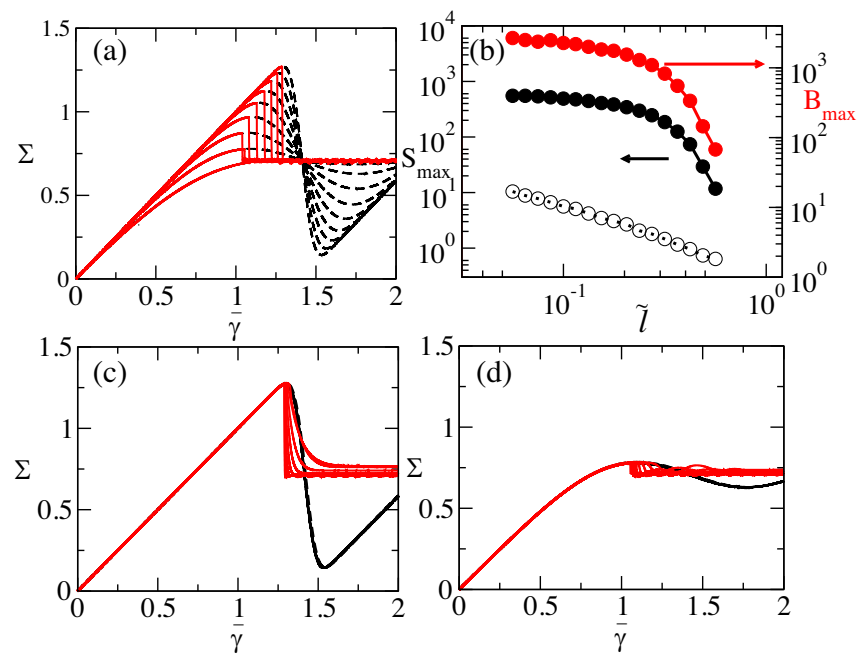

FIG. 4. (a) Stress vs strain in athermal elastoplastic model with homogeneous flow enforced (dashed lines) and shear banding allowed (solid lines). Shear rate $\overline{\dot{\gamma}}=10^{-4}$. Annealing increases with decreasing $\tilde{l}=0.562,0.421,0.316,0.237,0.177,0.133,0.1$, $0.0749,0.0562$ in curves left to right. (b) Left vertical axis: steepest negative slope in stress-strain curve with homogeneous flow enforced (open symbols) and allowing banding (closed black symbols) vs $\tilde{l}$, for fixed $\overline{\dot{\gamma}}=10^{-4}$. Right vertical axis: corresponding maximum degree of shear banding. (c),(d) curves in same format as (a), but now for fixed strong annealing $\tilde{l}=0.0562$ (c) or weak annealing $\tilde{l}=0.421$ (d) for imposed $\overline{\dot{\gamma}}=10^{-n}$ with $n=2.00,2.25, \ldots, 4.00$; steeper stress drop for smaller $\overline{\dot{\gamma}} . \eta=0.05, w=0.05, N=20, M=80000, D t=0.05$.

are shown by contours left to right. In this athermal case, even for the smallest accessible overshoot height, $B_{\max }$ and $S_{\max }$ increase without bound as $\overline{\dot{\gamma}} \rightarrow 0$, giving brittle yielding.

To understand these numerical results, we performed [39] a linear stability analysis showing that, in slow shear,
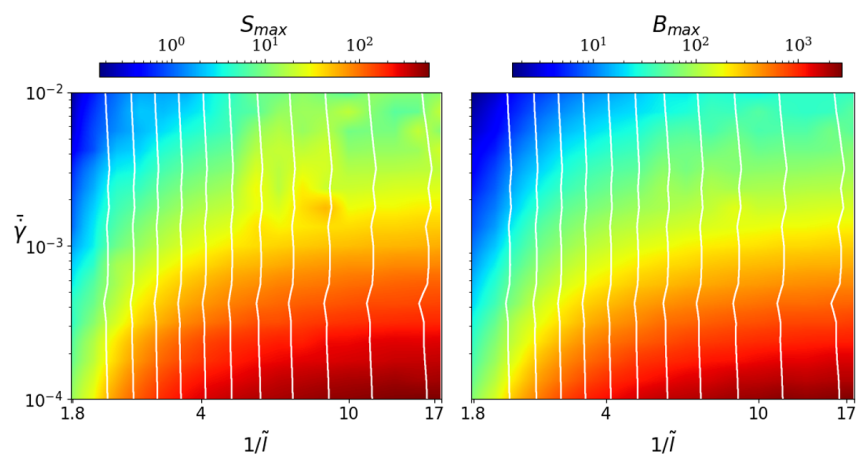

FIG. 5. Color scale showing in athermal elastoplastic model (left) steepest negative slope of stress versus strain $S_{\max }$ and (right) maximum degree of shear banding $B_{\max }$ during deformation. Each coordinate pair represents a deformation simulation with annealing parameter $1 / \tilde{l}$ and imposed shear rate $\overline{\dot{\gamma}}$. Contour lines show size of stress overshoot (peak minus steady state stress) $0.05,0.10, \ldots, 0.55$ from left to right. $\eta=0.05, w=0.05$, $N=20, M=80000, D t=0.05$. 
$\overline{\dot{\gamma}} \rightarrow 0$, the severity of shear strain banding $\delta \gamma(t)$ diverges as $\delta \gamma(0) / \partial_{\gamma} \Sigma$ on approach to the stress overshoot, $\partial_{\gamma} \Sigma=0$, indeed giving brittle failure, however small the stress overshoot, in this athermal case.

In Figs. 2 and 3 of [39], we explore yielding in the soft glassy rheology model in two different athermal protocols, finding the same scenario as in Fig. 4, with brittle yielding as $\overline{\dot{\gamma}} \rightarrow 0$, however small the stress overshoot.

In this Letter, we have studied the yielding of amorphous materials as a function of initial sample annealing prior to shear. We have shown thermal systems to display a continuous progression, with increasing annealing, from ductile yielding, in which the sample remains homogeneous, to brittle yielding, in which it becomes strongly shear banded. This progression arises from an increase with annealing in the size of an overshoot in the underlying stress-strain curve for homogeneous shear, which causes a shear banding instability that becomes more severe with increasing annealing. In contrast, slowly sheared athermal systems with a stress overshoot always show brittle yielding, however small the overshoot. How the thermal scenario crosses over to the athermal one at low temperatures remains an open question.

The scenarios put forward here differ notably from that proposed in mean field [32,33], in which ductile and brittle yielding are separated by a critical point, at which the underlying stress-strain curve for homogeneous shear undergoes a qualitative change in shape, as sketched in Fig. 1(a). To discriminate between the scenario proposed here and that in Refs. [32,33], it would be interesting to perform particle simulations that disallow banding, to access the shape of the underlying stress-strain curve for homogeneous shear. Indeed, simulations for small numbers of particles in Fig. 2D of Ref. [32] give an indication that the homogeneous stress-strain curve has the form sketched in Figs. 1(b) and 1(c). It further suggests that the smooth athermal yielding seen for modest stress overshoots in [32] may stem from finite size effects, or some difference between particle-elastoplastic-continuum models yet to be uncovered.

We thank Kirsten Martens, Romain Mari, and Matthieu Wyart for discussions; and the Centre for Doctoral Training in Soft Matter and Functional Interfaces (SOFI CDT), Durham University, EPSRC (EP/L015536/1) and Schlumberger Cambridge Research for funding.

[1] D. Bonn, M. M. Denn, L. Berthier, T. Divoux, and S. Manneville, Rev. Mod. Phys. 89, 035005 (2017).

[2] P. Coussot, Rheol. Acta 57, 1 (2018).

[3] D. Bonn and M. M. Denn, Science 324, 1401 (2009).

[4] P. Coussot, Soft Matter 3, 528 (2007).

[5] M. M. Denn and D. Bonn, Rheol. Acta 50, 307 (2011).

[6] T. C. Hufnagel, C. A. Schuh, and M. L. Falk, Acta Mater. 109, 375 (2016).
[7] A. L. Greer, Y. Q. Cheng, and E. Ma, Mater. Sci. Eng. R 74, 71 (2013).

[8] T. Divoux, C. Barentin, and S. Manneville, Soft Matter 7, 9335 (2011).

[9] T. Divoux, D. Tamarii, C. Barentin, and S. Manneville, Phys. Rev. Lett. 104, 208301 (2010).

[10] T. Divoux, D. Tamarii, C. Barentin, S. Teitel, and S. Manneville, Soft Matter 8, 4151 (2012).

[11] V. Grenard, T. Divoux, N. Taberlet, and S. Manneville, Soft Matter 10, 1555 (2014).

[12] T. Gibaud, C. Barentin, N. Taberlet, and S. Manneville, Soft Matter 5, 3026 (2009).

[13] T. Gibaud, D. Frelat, and S. Manneville, Soft Matter 6, 3482 (2010).

[14] T. Gibaud, C. Barentin, and S. Manneville, Phys. Rev. Lett. 101, 258302 (2008).

[15] A. Kurokawa, V. Vidal, K. Kurita, T. Divoux, and S. Manneville, Soft Matter 11, 9026 (2015).

[16] T. Sentjabrskaja, P. Chaudhuri, M. Hermes, W. C. K. Poon, J. Horbach, S. U. Egelhaaf, and M. Laurati, Sci. Rep. 5, 11884 (2015).

[17] C. A. Schuh, T.C. Hufnagel, and U. Ramamurty, Acta Mater. 55, 4067 (2007).

[18] P. K. Jaiswal, I. Procaccia, C. Rainone, and M. Singh, Phys. Rev. Lett. 116, 085501 (2016).

[19] I. Procaccia, C. Rainone, and M. Singh, Phys. Rev. E 96, 032907 (2017).

[20] J. Lin, T. Gueudre, A. Rosso, and M. Wyart, Phys. Rev. Lett. 115, 168001 (2015)

[21] C. Liu, E. E. Ferrero, K. Martens, and J.-L. Barrat, Soft Matter 14, 8306 (2018).

[22] G. P. Shrivastav, P. Chaudhuri, and J. Horbach, Phys. Rev. E 94, 042605 (2016).

[23] G. P. Shrivastav, P. Chaudhuri, and J. Horbach, J. Rheol. 60, 835 (2016).

[24] A. Wisitsorasak and P. G. Wolynes, Proc. Natl. Acad. Sci. U.S.A. 109, 16068 (2012).

[25] G. Parisi, I. Procaccia, C. Rainone, and M. Singh, Proc. Natl. Acad. Sci. U.S.A. 114, 5577 (2017).

[26] S. K. Nandi, G. Biroli, J.-P. Bouchaud, K. Miyazaki, and D. R. Reichman, Phys. Rev. Lett. 113, 245701 (2014).

[27] C. Rainone, P. Urbani, H. Yoshino, and F. Zamponi, Phys. Rev. Lett. 114, 015701 (2015).

[28] P. Urbani and F. Zamponi, Phys. Rev. Lett. 118, 038001 (2017).

[29] S. Aime, L. Ramos, and L. Cipelletti, Proc. Natl. Acad. Sci. U.S.A. 115, 3587 (2018).

[30] S. Aime, L. Ramos, J. M. Fromental, G. Prevot, R. Jelinek, and L. Cipelletti, Rev. Sci. Instrum. 87, 123907 (2016).

[31] E. D. Knowlton, D. J. Pine, and L. Cipelletti, Soft Matter 10, 6931 (2014).

[32] M. Ozawa, L. Berthier, G. Biroli, A. Rosso, and G. Tarjus, Proc. Natl. Acad. Sci. U.S.A. 115, 6656 (2018).

[33] M. Popović, T. W. J. de Geus, and M. Wyart, Phys. Rev. E 98, 040901(R) (2018).

[34] R. L. Moorcroft and S. M. Fielding, Phys. Rev. Lett. 110, 086001 (2013). 
[35] M. L. Manning, J. S. Langer, and J. M. Carlson, Phys. Rev. E 76, 056106 (2007).

[36] M. L. Manning, E. G. Daub, J. S. Langer, and J. M. Carlson, Phys. Rev. E 79, 016110 (2009).

[37] R. L. Moorcroft, M. E. Cates, and S. M. Fielding, Phys. Rev. Lett. 106, 055502 (2011).

[38] A. Nicolas, E. E. Ferrero, K. Martens, and J.-L. Barrat, Rev. Mod. Phys. 90, 045006 (2018).

[39] See Supplemental Material at http://link.aps.org/ supplemental/10.1103/PhysRevLett.125.168003 for a linear stability analysis for the initial onset of shear bands in the thermal fluidity and athermal elastoplastic models, a description of the SGR model, numerical results for yielding in the thermal and athermal regimes of the SGR model, and the effects of noise on yielding.

[40] P. Sollich, F. Lequeux, P. Hebraud, and M. E. Cates, Phys. Rev. Lett. 78, 2020 (1997).

[41] M. Vasoya, C. H. Rycroft, and E. Bouchbinder, Phys. Rev. Applied 6, 024008 (2016).

[42] J. Ketkaew, W. Chen, H. Wang, A. Datye, M. Fan, G. Pereira, U. D. Schwarz, Z. Liu, R. Yamada, W. Dmowski et al., Nat. Commun. 9, 3271 (2018).

[43] M. Singh, M. Ozawa, and L. Berthier, Phys. Rev. Mater. 4, 025603 (2020).

[44] We define the degree of banding at any time as the variance in shear rate across the sample. 